\title{
Géocarrefour
}

$92 / 3 \mid 2018$

L'élevage dans tous ses territoires (dans les pays développés)

\section{L'élevage en milieu urbain entre nature et nourriture}

Le cas des métropoles de Lyon et Grenoble

Urban breeding between nature and food The case of the metropolises of Lyon

and Grenoble

\section{Claire Delfosse et Adrien Baysse-Lainé}

\section{OpenEdition}

Journals

Édition électronique

URL : http://journals.openedition.org/geocarrefour/12239

ISSN : 1960-601X

Éditeur

Association des amis de la Revue de géographie de Lyon

Ce document vous est offert par Université de Cergy-Pontoise

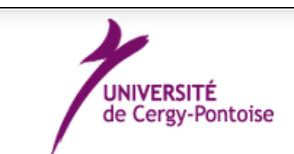

Référence électronique

Claire Delfosse et Adrien Baysse-Lainé, « L'élevage en milieu urbain entre nature et nourriture », Géocarrefour [En ligne], 92/3 | 2018, mis en ligne le 03 février 2019, consulté le 05 février 2019. URL http://journals.openedition.org/geocarrefour/12239

Ce document a été généré automatiquement le 5 février 2019

(c) Géocarrefour 


\title{
L'élevage en milieu urbain entre nature et nourriture
}

\author{
Le cas des métropoles de Lyon et Grenoble \\ Urban breeding between nature and food The case of the metropolises of Lyon \\ and Grenoble
}

Claire Delfosse et Adrien Baysse-Lainé

1 Dans les territoires métropolitains des pays du Nord, la présence de l'élevage est souvent oubliée ou méconnue (Delfosse et al., 2017), renvoyée aux seules périodes de crises nécessitant l'autoconsommation. Il est vrai que l'élevage en tant qu'activité agricole y est rarement dominant (Soulard et Thareau, 2009) et ses formes non agricoles (notamment récréatives) sont difficiles à repérer et, plus encore, à évaluer ${ }^{1}$. Pourtant l'élevage existe à la fois comme activité nourricière et sous des formes environnementales (éco-pâturage) dans les espaces tant urbains que périurbains. Il y répond à des enjeux renouvelés et prend de nouvelles formes qui interrogent le statut de l'animal d'élevage et ses fonctions.

2 Les débats récents sur l'approvisionnement alimentaire urbain, sur le statut des animaux dans les sociétés humaines (Porcher, 2011; Mouret, 2012; Gardin et al., 2018, Ayats et al, 2018) et sur les liens entre ville et nature réinterrogent la place de l'élevage dans les espaces fortement urbanisés. Les villes, en tant qu'espaces artificialisés, ont longtemps été considérées comme des espaces opposés à la nature. Aussi, face aux fortes demandes de nature, les acteurs urbains et en particulier métropolitains, cherchent à construire des villes «vertueuses» (Salomon-Cavin 2012). «La nature se pose donc toujours en opposition à la ville tout en en devenant une composante nécessaire » (Bourdeau-Lepage et Vidal, 2014), notamment en termes de bien-être (Bourdeau-Lepage, 2015). Elle l'est d'autant plus que la durabilité s'invite dans les débats sur l'aménagement et le développement des métropoles, tant pour ses liens à la «nature» que pour l'approvisionnement alimentaire et le lien social. À côté des espaces ouverts périurbains, les espaces verts urbains sont de plus en plus concernés par ces réflexions. Si depuis des décennies l'affirmation des liens ville-nature passait par la création d'espaces verts sans 
animaux (Blanc, 2003), la tendance s'inverse aujourd'hui et les espaces verts urbains «s'animalisent » de plus en plus.

Cet article a pour objet d'explorer les enjeux et rôles de l'élevage dans les espaces fortement urbanisés. Le propos est de donner à voir la diversité d'une activité généralement conçue comme exclusive de l'urbanité dense. Si les apports recherchés sont bien plus empiriques que théoriques, ils visent aussi à structurer des perspectives de recherche pour ce champ thématique encore relativement neuf, surtout dans les pays du Nord.

Pour ce faire, nous nous sommes appuyés sur les exemples des métropoles ${ }^{2}$ de Grenoble et de Lyon (carte 1), ce travail s'inscrivant dans le cadre du programme de recherche PSDR FRUGAL ${ }^{3}$. Le corpus est constitué d'entretiens semi-directifs menés avec des élus, des techniciens de collectivités locales, des éleveurs et des responsables d'association. L'analyse de la littérature grise ayant trait à l'agriculture des métropoles, de la presse agricole locale et des sites internet de collectivités territoriales et d'exploitations agricoles a complété les enquêtes.

\section{Carte 1 : Localisation des deux zones d'étude des métropoles de Lyon et Grenoble}

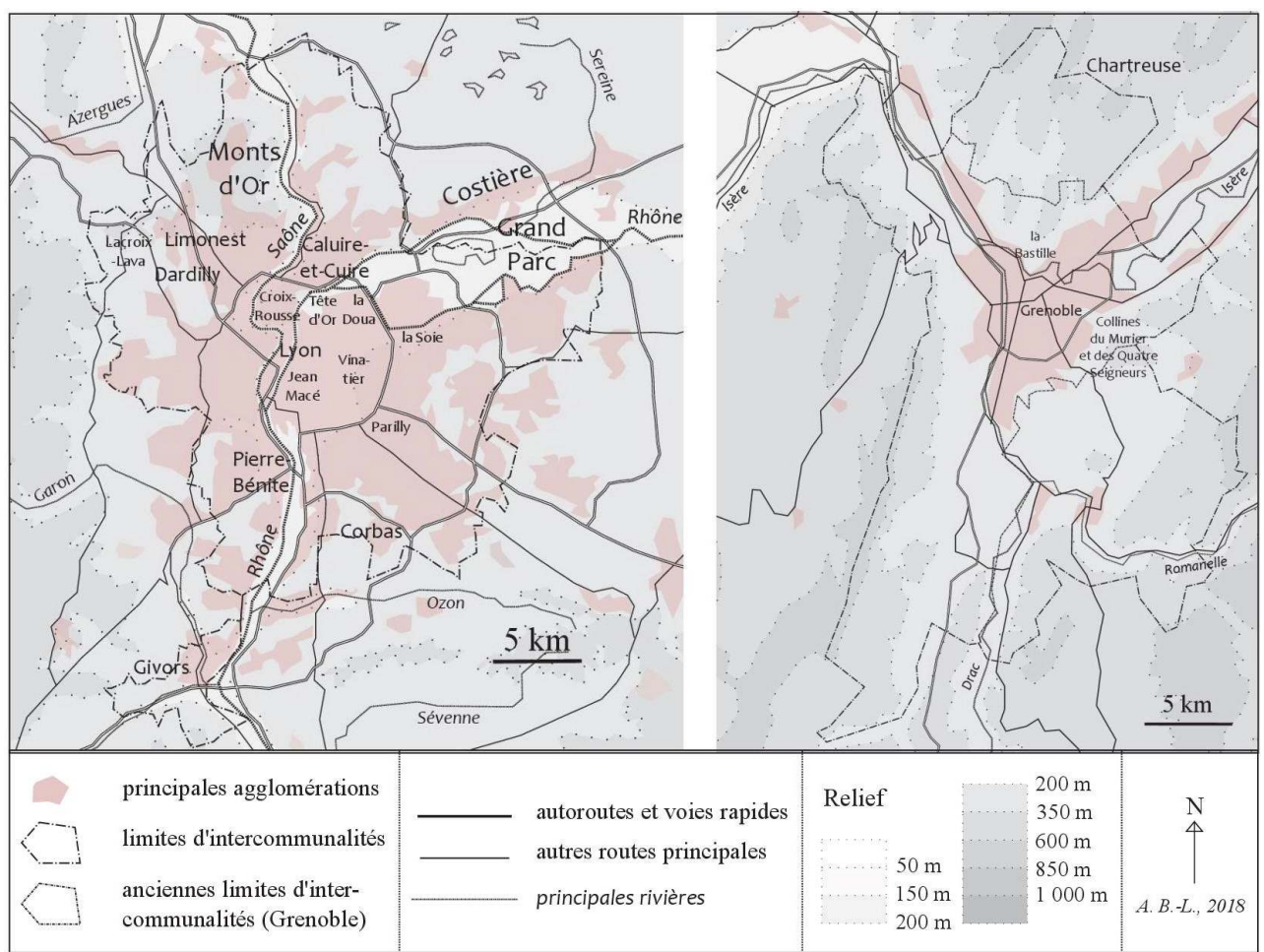

5 La situation et la place de de l'élevage agricole diffèrent d'une métropole à l'autre, aussi traiterons-nous dans un premier temps de chacune des villes séparément, notamment dans les fonctions assignées à l'élevage en termes de nature. Nous verrons ensuite dans quelle mesure et de quelle manière l'élevage s'invite dans les débats sur l'approvisionnement alimentaire urbain. L'article se clôt sur les tensions que les différentes fonctions et politiques peuvent susciter autour de l'élevage. 


\section{A Grenoble, l'élevage est une activité importante pour préserver le cadre de vie « naturel » et « montagnard »}

6 La métropole de Grenoble est une métropole montagnarde. Dans ce contexte, l'élevage joue un rôle important pour la préservation d'espaces ouverts considérés comme un cadre paysager attractif, tant pour résider que pour pratiquer des loisirs.

\section{Des exploitations d'élevage encore très présentes et qui entretiennent le paysage}

7 Dans la métropole grenobloise, sur les 210 exploitations agricoles (au sens du RGA) dénombrées en 2015 par les services de la collectivité, 144 d'entre elles font de l'élevage, dont la moitié en bovin. Il s'agit majoritairement d'exploitations à orientation viande, même si une grande diversité en termes de nombre et de type d'animaux (des bovins aux lapins et escargots), ainsi que de finalité de l'activité (produit alimentaire, loisirs), apparaît. Les exploitations d'élevage sont souvent non professionnelles au sens de la statistique agricole ${ }^{4}$, et qualifiées par les acteurs grenoblois de "patrimoniales». Les propriétaires-exploitants valorisent leur foncier par l'élevage, non pour avoir un revenu (lié aux ventes de viande), mais pour entretenir les parcelles afin d'éviter que la forêt ne gagne et que leur terrain ne soit dévalorisé : "Ils ont ainsi des vaches tondeuses ou des moutondeuses avec quelques revenus derrière mais qui restent minimes » (entretien Grenoble).

Les exploitations d'élevage, dans leur ensemble, revêtent un rôle très important car elles sont souvent le dernier rempart face à la déprise agricole, notamment sur les coteaux qui enserrent l'agglomération grenobloise. Ce rôle d'entretien de paysage ouvert est reconnu dans les politiques agricoles et d'urbanisme de la métropole, aux côtés de celui de gestion des risques naturels. Ainsi, dans le contrat vert et bleu ${ }^{5}$ de Grenoble Alpes Métropole 2017-2022, « un rôle essentiel d'accueil et d'ouverture au public » est confié à l'élevage des coteaux, lequel doit contribuer au bon fonctionnement du «poumon vert de la métropole». Pour encourager le maintien de l'élevage dans ces espaces, la métropole mène une politique d'aide à la réimplantation, l'implantation ou la modernisation de l'élevage (entretien Grenoble).

Cette politique a fait l'objet d'une action forte des acteurs métropolitains et contribué à la création d'une ferme intercommunale vouée à l'élevage sur la Colline du Murier et des Quatre Seigneurs. Considéré comme « un espace de respiration indispensable au cadre de vie et au bien-être des habitants de l'agglomération grenobloise " (Vargas, 2013), ce territoire a été protégé dans les documents de planification successifs. Toutefois, le recul de l'agriculture ne permettant plus d'entretenir cet espace de pentes, ce « site hautement stratégique et emblématique pour une agglomération 'nature' (ibid.), une démarche concertée entre les communes et l'intercommunalité, le Conseil régional ainsi que les habitants a été initiée. Dès les années 1990, des actions de préservation du paysage de ce site ont été mises en œuvre, et à partir des années 2000 s'affirme la nécessité de créer une ferme intercommunale. Il s'agit donc bien d'une opération visant à entretenir l'espace et à promouvoir les loisirs par une maitrise foncière, mais les collectivités territoriales impliquées sont allées plus loin et ont créé un siège d'exploitation. En effet, sur les espaces de pente, rares sont les sièges qui se maintiennent, obérant ainsi les possibilités 
d'installation-reprise d'exploitations d'élevage dites professionnelles. Le choix s'est porté sur l'élevage caprin car les chèvres sont de bonnes débrousailleuses, et le lait est transformé sur l'exploitation en produits laitiers.

10 La ferme a également une fonction pédagogique environnementale car sur cette colline se trouvait le centre d'éducation à l'environnement des villes de Saint-Martin-d'Hères et de Grenoble. Enfin, les éleveurs participent à des animations urbaines qui ont souvent un volet de découverte de la nature à laquelle est associée celle des animaux. Cette volonté de préservation d'une nature entretenue par l'agriculture est fortement affirmée sur le site internet de la Ferme des Maquis ${ }^{6}$. De fait, les procédures mobilisées dans le cadre de ce projet ont également relevé de l'aménagement agricole montagnard avec la création d'une association foncière pastorale (AFP), pour préserver le "caractère agricole vivant " d'un espace multifonctionnel qui favorise l'accueil des citadins et leurs loisirs. D'ailleurs un grand nombre d'exploitations agricoles de la métropole de Grenoble font de l'accueil, et l'on ne compte pas moins de trois fermes pédagogiques. Plusieurs exploitations sont regroupées au sein de l'association « des fermes buissonnières ", liée à la métropole ; elles se présentent comme " des fermes autour de Grenoble à visiter " ${ }^{7}$. La ferme du Maquis en fait partie et le chargé de mission de la métropole souligne qu'elle constitue pour lui un trait d'union entre la ville et la montagne (Vargas, 2013).

\section{L'élevage participe de la patrimonialisation de l'agriculture}

11 Par ailleurs, la ferme du Maquis participe de la mise en scène urbaine de l'agriculture, en particulier de l'agriculture de montagne vouée à l'élevage. La nouvelle gestion du fort de la Bastille (site fortement fréquenté qui domine le centre-ville) illustre cette volonté de valoriser l'élevage comme gestionnaire de la nature montagnarde, c'est-à-dire une nature marquée par l'étagement qui implique le mouvement des animaux. En effet, depuis 2016, le périmètre du fort est entretenu, pour le compte de la métropole, par un double actif renommé « berger de la Bastille » dans les documents de communication institutionnelle. Issu d'une commune de Chartreuse membre de la métropole, cet éleveur a accès en ville à des pâtures qu'il recherchait vainement en montagne. Ses allées et venues, accompagné de son patou et de son troupeau de moutons et de chèvres, sont l'occasion pour la ville de Grenoble d'organiser plusieurs animations à l'attention des habitants et des touristes. C'est une «fête de la transhumance » lors de l'arrivée et du départ du troupeau; ce sont des conférences sur le métier de berger, ainsi que des randonnées botaniques et folkloriques. Il s'agit de mettre en scène le pastoralisme, qui résonne dans l'esprit des Grenoblois car il correspond à l'idée de nature qu'ils se font, une nature synonyme de montagne (Tchékémian, 2016). D’ailleurs la métropole ne cesse de rappeler ses liens à la montagne et à l'élevage de montagne et insiste sur le fait que son territoire comprend désormais un alpage, depuis son extension récente vers les massifs avoisinant.

La patrimonialisation de la transhumance s'effectue en lien avec celle du pastoralisme et des produits alimentaires. Ainsi la venue de troupeaux sur les coteaux de la commune de Vaulnaveys-le-Haut est-elle qualifiée de mini-transhumance et fait l'objet de reportages de France 3 qui montrent les troupeaux de moutons et présentent les interviews d'habitants ou d'automobilistes. Tous soulignent l'attrait pour cette forme d'agriculture qui rappelle le passé. De même depuis 2010, une fête de la « Descente des alpages », initiée par un crémier fromager de Grenoble dont la boutique s'appelle "Fromagerie des alpages ", a lieu dans le centre de Grenoble. L'histoire agricole de la métropole, dont ont 
témoigné nombre d'expositions et de publications, fait également une large place à la production laitière y compris celle des ouvriers-paysans des coteaux qui avaient des vaches laitières et qui livraient leur lait à Grenoble ${ }^{8}$.

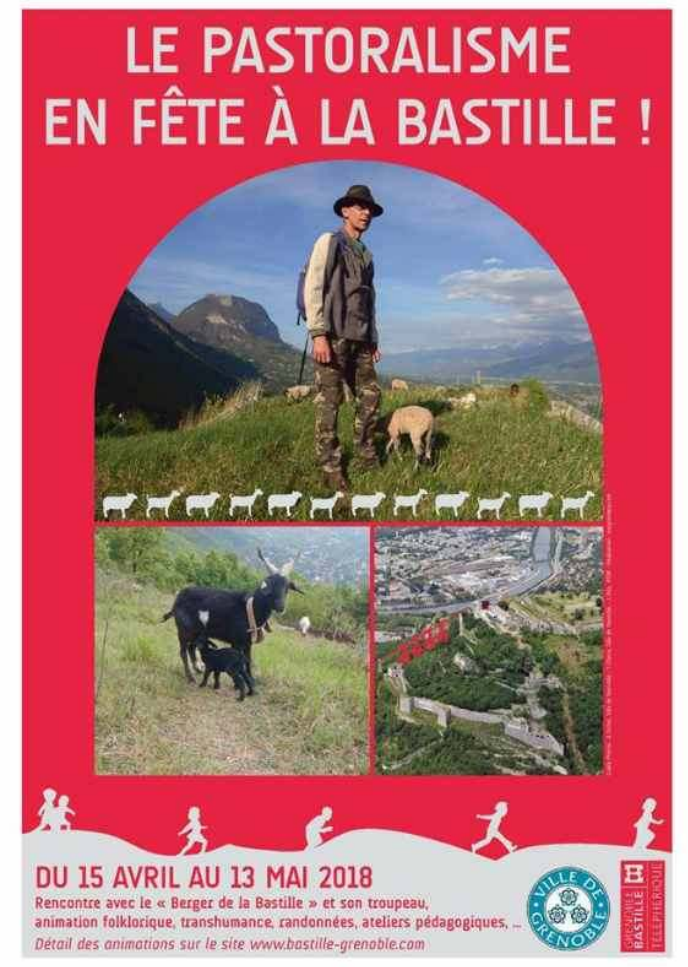

13 Ainsi à Grenoble l'élevage participe de la mise en valeur du paysage et du patrimoine agricole en lien avec la nature montagnarde. Il permet de réaffirmer les liens entre la métropole et les massifs alpins qui l'entourent.

\section{A Lyon, un élevage pour « renaturer » le territoire métropolitain?}

14 L'élevage est dans une situation différente à Lyon : il contribue à la renaturation de la métropole plus qu'il n'est un vecteur de découverte et d'ouverture vers une nature environnante. Dans un cadre plus fortement urbanisé qu'à Grenoble, l'élevage agricole tient une faible place, cantonné principalement dans les espaces interstitiels urbains. Dans le périmètre du SCoT lyonnais ${ }^{9}$, sur les 250 exploitations recensées par la collectivité, seules 24 sont considérées comme relevant de l'élevage (entretien Lyon). Celui-ci est repoussé aux marges du territoire métropolitain, notamment à l'ouest dans les Monts d'Or, au sud sur le plateau de Givors et dans la plaine de l'est, où subsistent des exploitations de polyculture-élevage. L'élevage est présenté par le SCoT comme un atout pour la protection du paysage des Monts d'Or : « seul l'élevage peut y être valorisé, en tant que producteur essentiel [de leur] paysage ». Toutefois, à l'échelle métropolitaine, l'élevage est surtout invoqué comme vecteur de réintroduction de la nature en ville, tant dans les espaces périurbains que dans le tissu urbain. 


\section{La ville qui protège la « nature »?}

15 La ville ne recrée pas seulement un «cadre naturel », elle prend soin de la nature, voire la restaure. C'est une fonction classique énoncée dans les mesures de Projets agroenvironnementaux et climatiques (PAEC) ${ }^{10}$ : dans les environs de Givors, les éleveurs sont encouragés à maintenir des prairies permanentes pour limiter l'érosion par coulées de boue, tandis que dans les Monts d'Or ils sont mobilisés pour limiter l'enfrichement. L'élevage permet également de restaurer et de gérer une partie des espaces du parc de Miribel-Jonage, dénommé « Grand parc métropolitain » depuis les années 2000. Créé à la fin des années 1960, ce parc, portion de plaine inondable du Rhône à l'amont de Lyon et aménagé pour des usages récréatifs et pour la protection de la ressource en eau du captage de Crépieux-Charmy, fait aussi appel à l'élevage. Il est géré par un syndicat mixte et une société publique locale, qui d'un côté met à disposition de deux éleveurs 24 ha de prairies et, de l'autre côté, gère en direct 100 ha de pelouses sèches (zonées Natura 2000) de prairies de fauche et de terres naturelles. Pour entretenir ces espaces, le parc possède depuis la fin des années 1990 un troupeau de trente vaches, complété depuis 2012 par trente chèvres.

L'élevage s'immisce également dans ces interstices que sont les espaces verts et contribue à leur gestion durable. C'est notamment le cas de l'éco-pâturage, pratique très développée au sein de la métropole lyonnaise par des acteurs de statuts divers. Des politiques sont menées aux échelles municipale et métropolitaine. La réintroduction par plusieurs municipalités de petits ruminants pour gérer les espaces verts est encouragée par la législation sur le non-recours aux pesticides en ville et la réduction de la pollution, y compris auditive. Les animaux permettent également d'entretenir des espaces difficilement mécanisables comme ceux entourant les forts. Dans un premier temps, ces communes ont fait appel aux entreprises présentes sur le territoire métropolitain et avec qui elles avaient l'habitude de travailler. En effet, à Lyon deux acteurs s'affirment : d'une part, une association à caractère environnemental qui conduisait des actions pédagogiques autour de la biodiversité pour la métropole, a acquis des animaux dans ce cadre et est aujourd'hui très reconnue pour l'éco-pâturage ; d'autre part, une entreprise d'entretien d'espaces verts qui a développé de l'éco-pâturage pour offrir un service adapté aux municipalités, aux entreprises et aux établissements publics. Dans un second temps, quelques communes ont elles-mêmes acquis leur propre troupeau. Par exemple, les 50 ha d'espaces verts de l'hôpital du Vinatier sont entretenus par un troupeau de «six chèvres alpines qui nous servent de débroussailleuses ${ }^{11}$, venues compléter un troupeau plus ancien de daims et de chèvres du Sahel. L'éco-pâturage est également pratiqué par des entreprises et des établissements publics, comme l'Université Lyon 1, sur son site de la Doua. D'autres actions sont conduites en faveur de l'élevage par les collectivités territoriales pour la gestion de leurs espaces naturels. Un cheval de trait est ainsi dédié au parc métropolitain de Lacroix-Laval, qui possède un espace forestier important, pour réaliser un débardage doux et pour la collecte des déchets sur le périmètre du parc.

17 Ces animaux ne remplacent pas seulement les pesticides et la tonte à moteur, mais offrent aussi des moyens de recyclage des déchets organiques ménagers qui correspondent aux nouveaux impératifs d'économie circulaire. Cette fonction est principalement invoquée par les municipalités qui encouragent l'adoption de poules par leurs habitants. Ainsi peut-on lire sur les sites internet des deux mairies de Caluire-et-Cuire et Pierre-Bénite 
qu'une poule consomme $150 \mathrm{~kg}$ de déchets par an et qu'elle économise $30 \%$ du contenu des poubelles: ainsi, "les traitements des déchets sont moins coûteux pour la collectivité et génèrent moins de pollution $»^{12}$.

18 Au-delà même d'une gestion environnementale, l'élevage peut contribuer à restaurer la nature en ville par la protection de la biodiversité domestique. Le milieu urbain apparaît dès lors comme un lieu d'accueil et de préservation de la nature, face à des campagnes où ne subsisterait plus la diversité animale rurale, qu'elle soit «lointaine» (moutons écossais, ou Soay), issue d'autres régions (race pie noire bretonne) ou de races plus locales ou régionales (chèvre du Massif central). En effet, la plupart des animaux qui sont utilisés pour l'éco-pâturage appartiennent à des races à petits effectifs ou menacées. Ces races suscitent certes la curiosité, mais disposent surtout de qualités nouvellement recherchées, alors qu'elles étaient considérées comme un handicap pour l'agriculture de rente. Dites rustiques, elles supportent le froid et la pluie et demandent peu de soins pour les mises-bas. Ainsi les brebis Soay sont-elles caractérisées comme "rustiques; elles n'ont pas besoin d'être tondues, et demandent très peu de soins vétérinaires" (entretien Lyon). Le parc de Miribel Jonage revendique son rôle dans la préservation biodiversité domestique : son troupeau est composé expressément de vaches pies noires bretonnes et de chèvres $d u$ Massif central. Il a notamment formalisé une convention avec l'association de défense de cette dernière race, pour participer à l'amélioration et à la diffusion du cheptel : de jeunes mâles caprins sont vendus à des particuliers, « pour entretenir une parcelle en pente dans leur jardin, notamment sur la Costière » (entretien Lyon).

Des associations vendent également des animaux des races ovines rares, qu'ils élèvent, à des particuliers. En effet, les animaux d'élevage font leur retour dans les jardins des particuliers de la ville et périurbains, pour les entretenir et les animer. Outre les chèvres et les ânes (voir article dans le même numéro), on peut trouver des moutons, des abeilles et des volailles. Les ouvrages et sites internet dédiés à ces dernières se multiplient. Le développement de la vente de poulaillers individuels illustre également ce renouveau de l'élevage de volaille amateur.

La préservation de la biodiversité animale et végétale est particulièrement revendiquée en matière d'abeilles. De nombreuses formes d'apiculture urbaine se développent, dans le sillage notamment du programme Urban Bees mené à Villeurbanne au début des années 2010. De nouvelles activités se créent en termes d'offre de service autour des abeilles : des agriculteurs vendent des essaims ou les louent à des particuliers et des entreprises du tissu urbain. Des ruches sont également disposées sur les toits des établissements publics, comme les lycées, la SNCF (nouvelle gare de Jean Macé à Lyon) ${ }^{13}$ et Grand Lyon Habitat. Le parc de Gerland à Lyon accueille même des ruchers pour les abeilles sauvages. La relation entre abeilles et villes est présentée comme doublement bénéfique: les abeilles maintiendraient la biodiversité végétale urbaine, tandis que l'absence de pesticides leur offrirait un meilleur habitat qu'à la campagne. La ville serait donc un haut-lieu de la nature, alors que dans les campagnes, les abeilles seraient en danger. La presse locale indique ainsi que, « depuis les toits de Grand Lyon Habitat, ces petites ouvrières, 'gentilles et très productives', ont tout le loisir d'aller butiner les fleurs du parc de la Tête d'Or, des squares et de tous les arbres d'alignements qu'elles ont sur leur chemin. Des flâneries qui, a priori, sont sans danger puisqu'à Lyon, on jardine sans utiliser de produits phytosanitaires. À l'abri des intoxications et pour l'instant des frelons tueurs, les abeilles lyonnaises se portent bien, ce qui n'est pas forcément le cas de leurs cousines qui vivent à la campagne $»^{14}$. La survie de colonies 
d'abeilles dans les espaces urbains denses requalifie ces derniers comme des espaces sains et relativement peu pollués.

21 Les animaux dans ces espaces jouent aussi un rôle dans la sensibilisation des citadins à la nature par le rapport au "vivant » qu'ils supposent d'autant que ce de rapport au vivant, au bien-être animal s'insinue désormais dans les pratiques de loisirs urbaines.

\section{L'élevage pour renouer avec le vivant et « faire campagne en ville »}

Les loisirs de proximité peuvent être considérés comme des vecteurs de reconnexion à la nature en ville, par le biais d'une attention renforcée au bien-être animal. Randonnées, promenade, équitation, mais aussi jardinage apparaissent en effet comme des arguments dans l'affirmation des espaces métropolitains comme espaces de nature. Ainsi peut-on lire dans la brochure Guide des sentiers nature, éditée par le Grand Lyon, que « une balade au bord d'un ruisseau, au cœur d'une forêt, à la découverte des rapaces ou de fleurs protégées... Pas la peine d'aller bien loin pour partir au vert : $40 \% \mathrm{du}$ Grand Lyon est constitué d'espaces naturels ou agricoles ». Les centres équestres, y compris ceux insérés dans le bâti urbain, insistent sur leurs liens à la nature. Ainsi celui de l'UCPA, voisin du nouvel hippodrome lyonnais depuis 2009 et situé au cœur du centre de loisirs du nouveau quartier de la Soie à Vaulxen-Velin, propose aux enfants de "partager la vie du centre équestre», de pratiquer le poney dans " une approche ludique, pédagogique et sécurisée, valorisant le respect de l'animal, dans un environnement naturel exceptionnel et une ambiance conviviale $»^{15}$. Les loisirs équestres participent donc de la sensibilisation au bien-être animal.

Les espaces verts et parcs apparaissent comme des lieux privilégiés du rapprochement avec la nature, et en particulier avec le vivant. Ainsi, la plupart des parcs urbains de la métropole hébergent des animaux d'élevage (moutons, chèvres, chevaux, poneys), voire même une mini-ferme au parc de Lacroix-Laval. Ces animaux ne sont plus là seulement pour l'exotisme, comme dans les parcs zoologiques ou au Vinatier (avec ses daims et chèvres du Sahel), mais pour proposer une gestion environnementale et comme facteur de lien social, voire pour "agricoliser » ces espaces. Ainsi des espaces dits verts sont désormais qualifiés de "prairies", comme au sein du programme de reconversion d'une ancienne caserne à Bron ${ }^{16}$.

La campagne, agricole du fait de ses animaux, s'insinue alors en ville. Un nouveau type de ferme s'affirme, les fermes dites d'animation pour lesquelles ce ne sont pas les visiteurs qui se rendent sur la ferme, mais les animaux de la ferme qui sont présentés en ville à l'occasion de manifestations festives ou sont insérées dans le tissu urbain à des fins pédagogiques. Depuis 2014, le conseil de quartier de la Croix Rousse porte un projet de ferme d'animation urbaine dont l'origine serait la suivante: " Peut-être est-ce l'urgence de sensibiliser l'ensemble de la population à l'écologie et au respect des êtres vivants et de leur environnement, qui nous a encouragé-es à travailler pour la mettre sur pied». La brochure présentant le projet s'intitule symptomatiquement « Le vivant en ville ».

Les animaux dans les jardins participent de cette volonté d'abolir les frontières entre urbanité et ruralité, une ruralité souvent assimilée à l'agriculture, mais une agriculture de loisirs, comme le notent Darribehaude et Gardon (2016) «l'agriculture semble cantonnée ici à une forme de loisir, pratique ludique et de redécouverte de la nature ». 


\section{Des loisirs avec les animaux pour le bien-être des citadins} peut aussi être un facteur de lien entre les citadins eux-mêmes. D'ailleurs, les politiques en direction de la nature affirment leur vocation à favoriser le lien social et partant à renforcer le bien-être des citadins (Bourdeau-Lepage, 2015). L'élevage y contribue. Ains est-il considéré comme facteur de lien entre les employés des espaces verts qui gèrent les animaux : «quand on a besoin de regrouper les moutons, toute l'équipe des espaces verts cuvre collectivement pour les attraper, c'est un moment de camaraderie » (entretien Lyon). Certains sites des mairies donnent des nouvelles des troupeaux et suscitent un dialogue avec les administrés. Ce rôle se retrouve autour du cheval qui fait son retour dans les espaces urbains pour remplir des missions de service public (Vial et al., 2015). À Lyon, la police municipale a, depuis le milieu des années 2010, une section équestre et le parc de MiribelJonage compte une brigade de gardes à cheval. Dans les deux cas, le cheval joue un rôle pour les missions coercitives mais est majoritairement utilisé pour des actions préventives, «de proximité et d'échange avec la population $»^{17}$. Les gardes à cheval du parc de Miribel expliquent qu'il s'agit également d'un moyen écologique et efficace pour se déplacer dans un espace dit naturel. Les moutons qui «nettoient » le parc de Parilly sont aussi mis à disposition de l'Institut départemental de l'enfance et de la famille (IDEF) voisin, pour favoriser le contact avec les enfants. Les chèvres «débrousailleuses » de l'hôpital du Vinatier ont aussi cette fonction. Elles sont nourries une fois par semaine par des adolescents en difficulté. Le rôle d'apaisement de l'animal est enfin invoqué pour les animaux des fermes d'animation qui interviennent dans les EHPAD (établissement hospitalier pour personnes âgées dépendantes) et, plus largement, des fermes thérapeutiques (Beiger, 2014).

Ainsi à Lyon la qualité de vie passe-t-elle par une forme de réconciliation avec la nature, que l'élevage pourrait favoriser. Cette réconciliation tient également à de nouveaux rapports à l'élevage par l'alimentation.

\section{L'élevage pour nourrir la ville?}

La recherche de durabilité des métropoles passe aujourd'hui notamment par une réflexion accrue sur l'alimentation. Les deux métropoles étudiées qui ont depuis plusieurs décennies une politique en faveur de l'agriculture périurbaine, à des fins de protection des espaces ouverts promeuvent aussi la diversification des exploitations et notamment la vente en circuits courts (Brand et Bonnefoy, 2011). Si la plupart des actions conduites sont surtout centrées sur les productions végétales, l'élevage n'est pas absent.

\section{Des animaux qui profitent de l'agricolisation des espaces naturels et urbains...}

L'agricolisation des espaces dits naturels est le fait de collectivités territoriales dont les politiques agricoles périurbaines favorisant le maintien d'espaces ouverts deviennent plus nettement des politiques qui visent à renforcer le rôle alimentaire de l'agriculture périurbaine. L'évolution des politiques conduites par le syndicat mixte des Monts d'Or en constitue le meilleur exemple. Fondé en 1996, il cherche à protéger son territoire de

Géocarrefour, 92/3 | 2018 
l'urbanisation et met en œuvre à partir de 2004 une politique d'acquisition foncière agricole. La vocation était initialement paysagère et environnementale, mais elle encourage aujourd'hui une agriculture de proximité. Le foncier acquis libre est utilisé pour conforter ou installer de jeunes exploitants engagés dans des modèles alternatifs, avec un fort « retour alimentaire pour le territoire » (entretien Lyon, 2016), y compris pour les produits animaux. Le syndicat est également propriétaire d'une infrastructure de transformation fromagère à la ferme de l'Ermitage. Enfin une diversification des produits, notamment de l'élevage, est recherchée : un éleveur bovin (dont la viande est vendue en cagette) et un éleveur caprin (dont les produits transformés sont écoulés par divers circuits courts) ont été installés. Un projet de création d'une exploitation de porc élevés en plein air (toujours pour les circuits locaux) permettrait de valoriser des terrains pentus de sous-bois appartenant au syndicat. Cette politique très coûteuse est difficilement reproductible dans tout le territoire de la métropole. Elle montre toutefois que les politiques environnementale et paysagère peuvent s'appuyer sur un secteur souvent décrié pour ses impacts environnementaux, lorsqu'il participe à l'approvisionnement urbain.

Il en est de même de la ferme du Maquis. Le lait des chèvres y est transformé en fromages qui sont vendus en circuits courts (à la ferme, sur les marchés de détail, dans les magasins de producteurs et d'autres commerces alimentaires de la métropole). La métropole de Grenoble fait une très large promotion de cette expérience qui fait l'objet de publications ou est présentée régulièrement dans les fiches d'expérimentation. Toutefois, là encore, l'expérience est difficilement reproductible, même si la ferme est économiquement rentable, car elle a nécessité beaucoup de concertation entre de nombreux acteurs et le processus de création a été long et coûteux. Aussi est-ce la seule exploitation d'élevage à maîtrise foncière et immobilière de la collectivité, les deux autres exploitations créées étant en productions végétales (entretien Grenoble).

31 Si les politiques des collectivités sont coûteuses, il reste que l'agricolisation gagne aussi les espaces urbains, notamment les jardins collectifs, les jardins particuliers et les fermes d'animation situées en ville. Là encore, la production maraîchère est surtout mise en avant mais l'élevage y a sa place. Ainsi le projet de ferme d'animation de la Croix Rousse fait référence à un passé "agricole » du quartier, un passé d'autoconsommation dans lequel les animaux étaient présents et avec lequel il s'agit de renouer : «l'Ouest du plateau de la Croix Rousse a une tradition de terres agricoles dans le cadre de congrégations religieuses ou des grandes propriétés privées, et jusqu'au milieu du XX ème siècle on pouvait y voir des vaches, moutons et chèvres $»^{18}$. La ferme d'animation urbaine a pour vocation de sensibiliser et de former à l'agriculture et à l'alimentation durable. Si ce projet pédagogique peut être apparenté aux fermes urbaines à vocation pédagogique parisiennes, les animaux ont, ici aussi, une fonction de production alimentaire. Il en est de même des élevages des poules composteuses qui sont aussi pondeuses. À Caluire et Cuire, on précise que : "c'est une première à Caluire: la ville offre deux poules à tout foyer désireux de recycler ses déchets... et de profiter d'œufs frais $»^{19}$. Les abeilles sont également là pour produire, ainsi le miel des abeilles du toit Grand Lyon habitat " a un petit goût de tilleul, mais c'est un miel toutes fleurs qui est produit en plein centre de la Part-Dieu ». Les collectivités territoriales encouragent également l'installation de ruches dans les jardins collectifs. Là encore «renaturer la ville » est lié à la production d'aliments urbains et donc à l'agriculture urbaine. dans la complexification de la politique agricole du parc de Miribel-Jonage. Les activités 
d'élevage de ce dernier se justifient dans un premier temps par une fonction patrimoniale et environnementale (paysage, qualité de l'eau et biodiversité domestique) et sont aidés à ce titre. Le troupeau en régie directe n'avait initialement aucune finalité alimentaire. Toutefois cette dernière prend de plus en plus de place dans la stratégie du parc qui a créé une marque «Les saveurs du parc » pour les produits des agriculteurs qui exploitent ses terres, ainsi qu'un magasin. Un des éleveurs participe avec ses chèvres et ses produits laitiers à des actions du Grand Parc de formation-sensibilisation à l'agriculture et à l'alimentation. «Il montre [au public] qu'il y a des parcelles agricoles sur le parc qui servent à nourrir. C'est le côté sensibilisation. On a un public très déconnecté de l'agriculture: les gens demandent, sont curieux. Ils ont l'image du fromage de chèvre qui vient de l'Ardèche. L'éleveur leur apprend les petits, le trèfle, la luzerne. ». Le parc lui-même commence à valoriser son élevage en régie "pour pérenniser le projet sans les subventions environnementales». Depuis 2014, la viande bovine est occasionnellement proposée et des tests ont été effectués pour développer une mini-filière. Pour les chèvres, un accord a été passé avec un éleveur de la Loire qui en fait de la viande. Le parc apparaît ainsi comme une vitrine pour la politique alimentaire métropolitaine, y compris en termes de produits d'élevage.

\section{...mais des produits de l'élevage relativement en marge de la dynamique de relocalisation}

Les travaux sur l'agriculture urbaine et périurbaine insistent tous sur l'importance de la demande alimentaire de proximité et l'opportunité qu'elle offre à l'agriculture métropolitaine (Aubry et Chiffoleau, 2009, Zasada, 2011, Brand et Bonnefoy, 2011). Tel est le cas des deux métropoles étudiées, où les élevages sont rarement intégrés dans des filières longues mais ont souvent un volet important de transformation, notamment en fromagerie et charcuterie. La proximité suscite l'évolution d'exploitations déjà existantes (installation d'atelier de transformation de lait à la ferme avec parfois atelier porcin pour valoriser le petit-lait, vente de viande en caissette à la ferme pour une exploitation située sur un axe routier très passager), ainsi que de nouvelles installations agricoles, comme celles de cochons en plein air où " tout est valorisé de la tête à la queue " (entretien Grenoble).

Les collectivités territoriales et en particulier les métropoles cherchent à encourager la relocalisation de l'alimentation pour répondre aux attentes de leurs habitants (réassurance par le proche) ainsi qu'aux obligations du Grenelle de l'environnement, en termes d'approvisionnement local et en bio des cantines. Aussi favorisent-elles les produits des élevages locaux pour les cantines. Un éleveur des Monts d'Or qui s'est reconverti dans la transformation et la vente directe a non seulement un magasin de produits laitiers, mais vend également ses yaourts pour des cantines scolaires (entretien). Néanmoins, pour l'approvisionnement en viande, de nombreux freins persistent en termes de quantité et de type de morceaux ( Le problème c'est que la cantine, ne prend que les quarts avant et il faut trouver à fournir les quarts arrière »).

Les politiques urbaines passent surtout par des actions en faveur des circuits de proximité ainsi que des politiques actives d'aides à l'installation ou à la mutation des élevages existants, notamment les encouragements à la labellisation en agriculture biologique et plus généralement dans le cadre de systèmes plus durables. La métropole grenobloise propose des aides bonifiées pour les exploitations dont la production est destinée aux circuits courts. Une vingtaine d'exploitations comportant de l'élevage a été aidée : des 
exploitations laitières avec transformation en fromage (vache, brebis, chèvre), mais aussi des exploitations productrices de viande: des vaches allaitantes aux exploitations porcines avec transformation, en passant par les lapins et la volaille (entretien Grenoble). La Métropole lyonnaise encourage le développement de l'élevage de volaille et de porcs en plein air pour répondre à la demande, sans beaucoup de succès toutefois. L'élevage à l'herbe, n'est pas toujours facile à développer dans le cadre d'un foncier contraint comme l'est celui des espaces fortement urbanisés. La transformation des élevages existants, notamment ceux qui sont en polyculture-élevage, semble aussi difficile (entretien Lyon).

Développer l'approvisionnement local réinterroge les infrastructures qui sont fortement fragilisées dans les espaces métropolitains, notamment les abattoirs. Dans les deux métropoles, les nouveaux abattoirs créés dans les années 1970 sont désormais surdimensionnés par rapport à la demande actuelle ou inadaptés. L'abattoir de Corbas, dans l'agglomération lyonnaise, pouvait produire 25000 tonnes de viande en 1977, alors qu'il n'en produit que 6000 actuellement (Hurstel, 2018). L'abattoir de Grenoble fait l'objet d'une modernisation-adaptation, notamment pour l'approvisionnement local : « Les filières animales pour nous c'est très important. On investit dans l'abattoir c'est un gros budget pour la métropole et le département. Il y a une salle de découpe. C'est un outil d'abattage et cela aide les bouchers du territoire" (entretien Grenoble). Ces investissements doivent également aider les éleveurs qui vendent en direct. L'abattoir de Corbas offre cette prestation de service pour quelques éleveurs bovins, caprins et ovins, mais l'abattage des volailles et des cochons n'est pas réglé sur l'agglomération lyonnaise. L'abattage est en effet une question sensible dans ces territoires, et cela particulièrement pour l'Aïd, l'abattage dit illégal posant des problèmes sanitaires (entretien Lyon). Ainsi trois éleveurs des environs de Givors se sont-ils organisés, aidés par les collectivités territoriales, pour créer un abattoir mobile pour l'Aïd.

Pourtant, les politiques alimentaires urbaines ne sont pas toujours favorables à l'élevage. L'objectif de relocalisation, voire d'autonomie, alimentaire oriente plus les collectivités territoriales vers les productions végétales, moins demandeuses de surfaces. C'est le sens, par exemple, des essais de constitution d'une filière courte en céréales (pain et pâtes) et légumineuses à partir des terres du Grand Parc. Le nombre d'agriculteurs boulangers recensés dans les deux métropoles et le succès qu'ils rencontrent illustrent cette attente (Buyck et Courcoux, 2019). Près du Grand Parc, les communes de Vaulx-en-Velin et Décines-Charpieu cherchent à développer le maraîchage dans une plaine désormais céréalière, en faisant référence à l'histoire de la zone maraîchère d'après-guerre, tout en oubliant la période antérieure où la même zone était dédiée à l'élevage laitier pour l'approvisionnement de l'agglomération lyonnaise (Baysse-Lainé, 2018). Les collectivités visent également à réduire la consommation de viande dans les cantines pour des arguments environnementaux: elle est le type d'aliment le plus gaspillé et dont la production nécessiterait le plus de surfaces agricoles, à calories égales. Il reste que les collectivités territoriales n'excluent pas la consommation de viande, mais visent une viande de qualité qui rend des services environnementaux, «par le recours aux prairies par exemple ( (entretiens Lyon et Grenoble).

L'élevage occupe un entre-deux dans l'alimentation relocalisée des villes : désiré et promu par les collectivités à l'étape de production, il est toutefois peu pris en charge aux autres étapes des chaînes alimentaires. Cette tension n'est pas la seule qui traverse le retour de l'élevage dans les espaces urbanisés. 


\section{L'élevage en tensions : voisinages, foncier et statut des animaux}

39 La multiplicité des fonctions et rôles dévolus à l'élevage suscite des tensions. Ces dernières tiennent aux représentations sociales de l'élevage et de la nature, ainsi qu'aux différents statuts des animaux dans notre société (Digard, 1998).

\section{L'élevage entre rêve et réalité}

L'élevage dans les espaces urbanisés n'échappe pas aux contradictions qui opposent une agriculture patrimonialisée (paysage de bocage, de prairies animées par des animaux d'élevage) mise en scène par les habitants, voire par les élus, et la réalité de l'activité économique agricole (Poulot et Rouyres, 2007). La première source de malentendus et de conflits est relative aux aménités, notamment paysagères, des espaces périurbains. Certains habitants s'opposent à la création de bâtiments d'élevage : ainsi que l'identifie l'Agence d'urbanisme de Lyon dans son diagnostic agricole de la métropole, « un grand bâtiment heurte les imaginaires ", alors que ce type de construction est particulièrement important pour l'élevage. En outre, l'aménagement des prairies des Monts d'Or pour le pâturage constant de chevaux génère des plaintes de résidents rebutés par la présence de vieilles baignoires ou de cabanes disgracieuses. De plus, les odeurs et effluents sont considérés comme des nuisances par les habitants, notamment dans le parcellaires particulièrement mité et émietté, où des animaux paissent à proximité immédiate des habitations. Un éleveur de chèvres de Limones dû mettre en place « un protocole de lutte contre les mouches » (entretien Lyon) face aux envois d'huissier d'un collectif de voisins. Les nuisances liées à l'élevage contribueraient aussi à dévaloriser le bâti proche (entretien Grenoble). Les élevages avicoles et porcins sont encore plus décriés que d'autres, même dans le cas du projet porcin de plein air dans les Monts d'Or.

41 Les tensions entre rôle rêvé et réalité de l'élevage se retrouvent pour l'éco-pâturage, autour du degré de domestication des animaux. La gestion des animaux dans les espaces verts n'est pas sans contraintes et difficultés, surtout lorsque les animaux ne sont pas conduits par un berger. Des agents municipaux doivent alors éviter le surpâturage, gérer les mises bas, tout en étant bien moins disponibles temporellement que des éleveurs. Même dans les cas où les naissances ne posent pas de problème, comme pour la race Soay, il faut veiller à éviter la consanguinité, d'où des échanges entre propriétaires de troupeaux. Ces contraintes, que les gestionnaires des espaces verts doivent prendre en compte, ont aussi un aspect positif. Pour le personnel communal, la responsabilité de la surveillance d'un troupeau constitue une reconnaissance de compétence, distincte de la gestion des machines et des produits phytosanitaires. Par ailleurs, certains acteurs de l'éco-pâturage mettent en avant le caractère sauvage, presque non-domestique, de leurs animaux. Il s'agit de garantir la vocation d'entretien de l'espace naturel : les éleveurs auraient trop tendance à nourrir leurs bêtes et ces dernières « nettoieraient moins le milieu » (entretien Lyon). Enfin, le caractère sauvage des moutons Soay les protégerait du vol ou d'une mort donnée " par jeu ou par désœuvrement " par des habitants (entretien Lyon).

L'élevage d'animaux par les particuliers ne s'improvise pas non plus. Aussi les collectivités territoriales élaborent-elles des cahiers des charges et obligent-elles les familles qui adoptent des poules à se former ${ }^{20}$. Malgré cela, ces tentatives ne se soldent 
pas nécessairement par des succès : à Lyon, l'hôpital du Vinatier reçoit ainsi des poules dont les particuliers ne veulent plus. Cet élevage nécessite aussi que les collectivités territoriales revoient ou réinterrogent leurs réglementations. Les nouvelles fonctions des animaux (présence ornementale dans les jardins, care dans les hôpitaux, pédagogique dans les fermes d'animation) suscitent des interrogations sur le bien-être animal (Darribehaude et al., 2016) et sur la gestion de la fin de vie des animaux.

\section{Des animaux à manger ?}

43 animaux dans ces espaces et la vocation nourricière qui peut leur être donnée. A Paris, les bergers urbains insistent sur le rôle alimentaire des animaux utilisés pour l'éco-pâturage, mais tel n'est pas le cas dans la métropole lyonnaise. La valorisation en viande peut apparaitre incompatible avec le lien social fourni par les animaux. Les perceptions diffèrent selon les entreprises d'éco-pâturage. De même il n'est pas question de manger les animaux de la future ferme d'animation de la Croix Rousse. Comme le note Hirschler (2016), "personne [au sein du comité de préfiguration] n'est favorable à l'élevage hors-sol et personne ne m'a suggéré d'élever des animaux à viande. La mort de l'animal a au contraire suscité de vives réactions d'oppositions ». Par ailleurs, les animaux élevés dans les jardins des particuliers deviennent parfois des animaux de compagnie, les poules obtenant peu ou prou le statut des chiens.

Dès lors, il apparait plus facile pour l'élevage agricole de valoriser et promouvoir les produits laitiers que la viande.Comme le souligne la monographie agricole de la métropole de Grenoble, la filière viande souffre d'un "manque de reconnaissance dans une agglomération qui soupçonne peu son existence. Pourtant c'est une forme d'agriculture périurbaine incontournable pour l'entretien du paysage de l'agglomération notamment $»^{21}$. Les actions festives en faveur de l'agriculture peuvent sensibiliser les habitants à la réalité de l'agriculture et à ses enjeux alimentaires. Ainsi le Syndicat mixte des Monts d'Or a-t-il coorganisé avec l'institut Sandar de Limonest, une école privée d'agriculture, la première « Fête de l'agriculture des Monts d'Or " à l'automne 2018. Encore une fois, la fabrication de fromage et de produits laitiers est plus facile à mettre en valeur dans de tels événements. Il en est de même dans le cadre du Grand Parc, où c'est l'élevage de chèvres qui fait l'objet de mise en scène et d'actions pédagogiques et pas celui de veaux, comme dans les fermes pédagogiques de la métropole de Grenoble. Il reste que quelques exploitations organisent tout de même, par exemple, des stages autour de la fabrication de charcuterie pour les adultes : la ferme Montgardier les présente comme des sessions de formation pour « découvrir, apprendre, mettre en pratique nos recettes et notre savoirfaire pour passer le cochon du champ à l'assiette $»^{22}$.

Une dernière tension est interne aux politiques métropolitaines. À Lyon, elle oppose le développement des circuits relocalisés et la promotion de la gastronomie traditionnelle de la ville, qui repose largement sur des produits carnés souvent plus régionaux que locaux. À Grenoble les fromages, et plus généralement les produits laitiers, sont valorisés parce que produits dans les montagnes proches, mais il est difficile de se procurer du beurre et des produits laitiers frais de proximité. En matière de produits gastronomiques, la ville joue sur la complémentarité avec les territoires voisins qui sont des PNR. Dans les deux cas, la promotion des produits passe par une politique de complémentarité entre territoires métropolitains et territoires voisins, une politique qui pourrait aussi à terme, 
notamment pour Lyon, entériner le fait que l'élevage continue à reculer sur le territoire métropolitain. Les cantines de la métropole acceptent d'ailleurs de s'approvisionner en produits carnés provenant de plus loin (échelle régionale) que les produits végétaux (échelle locale).

\section{Un foncier à partager entre élevages récréatif et agricole}

Une tension foncière importante existe entre l'élevage voué aux loisirs, notamment l'élevage équin, et l'élevage de rente. Le cheval tient une place particulière dans les espaces périurbains et tend même à devenir un indicateur de périurbanité . Il est le support d'entreprises équines comme les centres équestres, qui relèvent désormais du statut agricole, ou peut constituer une forme de diversification pour d'autres exploitations agricoles. Ainsi à Grenoble 44 exploitations ont des chevaux dont 25 au titre de l'activité principale, qui incluent 15 poneys clubs, centres équestres et pensions spécialisés dans les gros effectifs. «A peu près $15 \%$ des exploitations ont développé des ateliers de pensions de chevaux pour les citadins propriétaires de chevaux de loisirs" (entretien Grenoble). En effet, alors que certains particuliers s'installent en milieu périurbain pour disposer de terrains et bâtiments adaptés à leurs animaux, d'autres offrent le prix fort pour mettre leurs chevaux en pension. Dans les Monts d'or, un mois d'une telle location correspond à un an de location agricole en fermage. Comme le souligne la boutade d'un agriculteur, "dès qu'il y a une vache qui s'en va, il y a deux chevaux qui arrivent " (entretien Lyon). Certaines collectivités s'inquiètent de cette évolution et cherchent à favoriser les usages agricoles par le biais foncier, comme le syndicat mixte des Monts d'Or du Lyonnais et la métropole grenobloise. Dans ces contextes, les centres équestres se sentent exclus : des tensions existent notamment entre le syndicat mixte des Monts d'Or et des entreprises de promenade équine soutenues par des exploitants non équins historiques du massif.

De même il peut y avoir des tensions entre les exploitations dites patrimoniales, récréatives, qui certes jouent un rôle d'entretien, et peuvent contribuer à de la production d'aliments (y compris parfois en circuit courts avec la vente de viande en caissettes), mais peuvent freiner ou bloquer de nouvelles exploitations d'élevage «professionnelles».

\section{Conclusion}

Notre analyse des place, rôles et fonctions de l'élevage dans les métropoles de Lyon et Grenoble a permis de montrer l'intérêt de l'étude d'un objet trop souvent invisibilisé dans la littérature. À Grenoble, l'élevage est mis en avant par les acteurs publics pour préserver un cadre paysager de pâtures montagnardes. Ses animaux sont le support d'une mise en scène du lien de la ville à la nature et d'une patrimonialisation de l'agriculture. À Lyon, l'élevage n'est pas tant un révélateur de l'environnement non artificialisé qu'un vecteur de renaturation de la ville. Par l'intermédiaire de l'éco-pâturage, de la réintroduction de poules ou de l'accueil de colonies de ruches, l'espace urbain se présente comme le conservatoire d'une biodiversité domestique que les campagnes peinent à préserver. Prenant soin d'une nature qu'elle participe à construire, la ville accueille des animaux dédiés au bien-être et à la récréation des citadins. Au-delà de ces deux types de relations à l'élevage et à ses animaux, les métropoles de Grenoble et Lyon ont en partage un intérêt 
affirmé pour le développement des circuits alimentaires locaux. L'élevage est mobilisé dans le cadre des stratégies publiques d'installation agricole et de maintien de la production près des villes, ce qui constitue une originalité par rapport à de nombreuses collectivités françaises qui se limitent au maraîchage. Les contraintes propres à la mise en marché des produits carnés (équilibre des morceaux, infrastructure) impliquent toutefois que ce sont plutôt les produits laitiers qui répondent aux demandes d'alimentation locale. Des contradictions apparaissent également entre la préservation du patrimoine gastronomique et l'évolution des régimes alimentaires. Plus largement, des tensions se font jour dans trois domaines: la localisation des animaux dans le tissu urbain et périurbain, le degré de domestication de ces animaux, la coexistence de plusieurs modèles d'élevage. Ces thématiques méritent d'être approfondies car elles rejoignent des enjeux fondamentaux de l'aménagement des territoires, à savoir la gestion du foncier, l'évolution du statut social des animaux et l'adéquation de l'activité agricole aux demandes sociales en fort développement.

Enfin, le choix de deux villes situées à proximité de zones herbagères pour conduire les enquêtes n'est pas sans conséquence sur l'évaluation de la place et du rôle de l'élevage. Les villes situées au sein des bassins céréaliers, par ailleurs en expansion, doivent probablement être moins marquées par le retour des animaux et de l'élevage. De fait, le mouvement social et entrepreneurial particulièrement dynamique de l'agriculture urbaine est largement structuré autour des végétaux et laisse peu de place aux animaux. Au-delà des approfondissements thématiques évoqués ci-dessus, il importe donc de comparer désormais nos résultats rhône-alpins avec de nouvelles enquêtes conduites dans d'autres villes et ainsi d'approfondir les tensions existantes autour de l'agriculture urbaine entre préservation environnementale et injonction alimentaire, sans oublier les débats sociétaux autour de l'élevage.

\section{BIBLIOGRAPHIE}

AYATS J.-F., DELFOSSE C., PLUVINAGE J., PORCHER J. (dir.), 2018, Éloge de l'élevage... Mais quel élevage ?, Pour, $\mathrm{n}^{\circ} 231$.

ALAVOINE MORNAS F., GIRARD S., 2014, Foncier agricole et enjeux environnementaux : de nouvelles tensions au niveau des exploitations agricoles pour la mise en oeuvre de la trame verte, communication présentée au colloque Le foncier agricole. Usages, tensions et régulations, 11-12 juin 2014, Lyon.

AUBRY C., CHIFFOLEAU Y, 2009, Le développement des circuits courts et l'agriculture périurbaine : histoire, évolution en cours et questions actuelles, Innovations agronomiques, $\mathrm{n}^{\circ} 5$, p. 53-67.

BANZO M., VALETTE E., 2006, Les espaces non bâtis, enjeux de nouvelles pratiques et de nouveaux vécus urbains, Sud-Ouest Européen, n²2, p. 27-39. 
BAYSSE-LAINE A., 2018, Terres nourricières ? La gestion de l'accès au foncier agricole en France face aux demandes de relocalisation alimentaire, thèse de doctorat en géographie et aménagement, Université de Lyon.

BEIGER F., 2014, Exemples de lieux thérapeutiques, in BEIGER F. (dir.) Eduquer avec les animaux. La zoothérapie au service des jeunes en difficulté, Paris, Dunod, p. 89-104.

BLANC N., 2003, La place de l'animal dans les politiques urbaines, Communications, $\mathrm{n}^{\circ} 74$, p. $159-175$.

BOURDEAU-LEPAGE L., 2015, La nature en question. Aménager en ménageant l'homme et son milieu, Géocarrefour, vol. 90, n³, p. 191-192.

BOURDEAU-LEPAGE L., VIDAL R., 2012, Nature urbaine en débat : à quelle demande sociale répond la nature en ville?, Déméter, p. 195-210.

BOURDEAU-LEPAGE L., VIDAL R, 2014, Nature en ville : attentes citadines et actions publiques, Paris, Editopics, $120 \mathrm{p}$.

BRAND C., BONNEFOY S., 2011, L'alimentation des sociétés urbaines : une cure de jouvence pour l'agriculture des territoires métropolitains ?, VertigO, nº 11, DOI : 10.4000/vertigo.11199

BUYCK J., COURCOUX T., 2019, Economies, spatialités et urbanités du pain : explorations dans la métropole grenobloise, in X. GUILLOT, P. VERSTEEGH, Transitions économiques et nouvelles ruralités, Presses universitaires de Saint-Etienne, à paraître.

CHALAS Y., 2005, La ville-nature contemporaine. La demande habitante à l'Isle-d'Abeau, Annales de la Recherche urbaine, $\mathrm{n}^{\circ}$ 98, p. 43-49.

DARLY S., 2014. Des moutons dans la ville : quelles externalités environnementales des pratiques d'élevage ovin en milieu urbain ?, Pour, n² 224, p. 285-290.

DARRIBEHAUDE F., GARDON S., 2016, Quelle agriculture dans la ville ? Rétrospective et perspectives dans l'agglomération lyonnaise ", in F. DARRIBEHAUDE, S. GARDON, B. LENSEL (dir.), Le vivant en ville. Nouvelles émergences : gouvernance, intensité urbaine, agriculture et animalité urbaines, Lyon, Métropole de Lyon, 224 p., p. 146-153.

DELFOSSE C., 2016, La valorisation de la biodiversité domestique cultivée, une nouvelle ressource pour les espaces ruraux ?, p. 213-225 in E. GLON, B. PECQUEUR (dir.), Au coeur des territoires créatifs ? Proximités et ressources territoriales, Rennes, PUR, 296 p.

DELFOSSE C., DUMONT B., HOSTIOU N., 2017, Des services contrastés rendus par l'élevage dans les espaces urbains et périurbains européens, Productions animales, vol 30, n 4, p. 395-406.

DIGARD J.-P., 1998, Les Français et leurs animaux, Paris, Fayard.

FLEURY A., DONADIEU P., 2033, La construction contemporaine de la ville-campagne en Europe, Revue de géographie alpine, vol. 91, $\mathrm{n}^{\circ}$ 4, p. 19-29.

GARDIN J., ESTEBANEZ J., MOREAU S., 2018, Comme la biche tétanisée dans les phares de la bagnole : la justice spatiale et les animaux, justice spatiale / spatial justice, $\mathrm{n}^{\circ} 12$, https:// www.jssj.org/article/comme-la-biche-tetanisee-dans-les-phares-de-la-bagnole-la-justicespatiale-et-les-animaux/

GERMAIN P., THAREAU B., 2011, Les agriculteurs face à l'urbanisation II. Garantir la pérennité des exploitations, Études Foncières, $n^{\circ}$ 150, p 19-23.

HERCULE J., CHATELLIER V., PIET L., DUMONT B., BENOIT M, DELABY L., DONNARS C., SAVINI I., DUPRAZ P., 2017, Une typologie pour représenter la diversité des territoires d'élevage en Europe, Productions animales, vol. 30, $\mathrm{n}^{\circ}$ 4, p. 285-302. 
HIRSCHLER S., 2016, Une ferme d'animation sur la Croix-Rousse ? État des lieux sociologique et suggestions pour la gouvernance de ce projet, mémoire de master en sociologie, Université Lumière Lyon 2.

HURSTEL L., 2018, Commercialisation des produits carnés à Lyon. Évolution et adaptation des grossistes et détaillants face aux changements, mémoire de master en géographie, ENS de Lyon.

MOURET S., 2012, Élever et tuer des animaux, Paris, PUF, 224 p.

PORCHER J., 2011, Vivre avec les animaux. Une utopie pour le XXI ${ }^{e ̀ m e}$ siècle, La Découverte.

POULOT M., 2013, Du vert dans le périurbain. Les espaces ouverts, une hybridation de l'espace public (exemples franciliens), EspacesTemps.net, http://www.espacestemps.net/articles/du-vertdans-le-periurbain-les-espaces-ouverts-une-hybridation

POULOT M., ROUYRES T., 2007, Refaire campagne en Île-de-France, Norois, n² 202, p. 61-71.

SALOMON-CAVIN J., 2012, Entre ville stérile et ville fertile, l'émergence de l'agriculture urbaine en Suisse, Environnement urbain/Urban environment, $\mathrm{n}^{\circ}$ 6, p. 17-31.

SOULARD C.-T., THAREAU B., 2009, Les exploitations agricoles périurbaines : spécificités et logiques de développement, Innovations Agronomiques, $n^{\circ}$ 5, p. 27-40.

TCHEKEMIAN A., 2016, La nature de la ville. Les représentations sociales des éléments de nature à Grenoble et leurs usages par les institutions d'aménagement et d'animations, in $\mathrm{F}$.

DARRIBEHAUDE, S. GARDON, B. LENSEL (dir.), Le vivant en ville. Nouvelles émergences : gouvernance, intensité urbaine, agriculture et animalité urbaines, Lyon, Métropole de Lyon, 224 p., p. 112-121.

VARGAS L., 2013, La ferme intercommunale du Maquis, Pour, nº 220, p. 163-171.

VIAL C., AUBERT M., PERRIER-CORNET P., 2011, Les choix organisationnels des propriétaires de chevaux de loisir dans les espaces ruraux, Économie rurale, $\mathrm{n}^{\circ} 321$, p. 42-57.

VIDAL R., FLEURY A., 2009, La place de l'agriculture dans la métropole verte. Nostalgies, utopies et réalités dans l'aménagement des territoires aux franges urbaines, Projets de paysage, http:// www.projetsdepaysage.fr/la_place_de_l_agriculture_dans_la_metropole_verte

ZASADA I., 2011, Multifunctional Peri-Urban Agriculture. A Review of Societal Demands and the Provision of Goods and Services by Farming, Land Use Policy, vol. 28, nº 4, p. 639-648.

\section{NOTES}

1. Peu d'entrées du Recensement général agricole (RGA) permettent de rendre compte de la place de l'élevage : le cheptel est présenté de manière agrégée en UGB (« unité de gros bétail », dont l'étalon est peu ou prou une vache laitière adulte), sans distinction des espèces; les surfaces en prairies permanentes sont indiquées sous la dénomination de "surfaces toujours en herbe », ce qui ne renvoie toutefois qu'aux activités d'élevage herbager.

2. Le terme est ici entendu dans son acception administrative : nous nous sommes limités à la collectivité territoriale à statut particulier du Grand Lyon (dite Métropole de Lyon) et à l'intercommunalités de Grenoble Alpes Métropole.

3. Pour et Sur le Développement Régional (www.psdr.fr) FoRmes Urbaines et Gouvernance ALimentaire (http://projetfrugal.fr/). Ce programme est notamment conduit dans l'ancienne région Rhône-Alpes.

4. C'est-à-dire qu'elles génèrent une marge brute trop faible ou que trop d'heures de travail y sont comptabilisées. 
5. Mis en œuvre par l'ancienne région Rhône-Alpes les contrats verts et bleus visent à maintenir et à restaurer des trames vertes et bleues à l'échelle de territoires de projet.

6. http://www.fermedesmaquis.fr/

7. http://les-fermes-buissonnieres.fr/

8. Voir par exemple : Musée dauphinois, 1993, Grenoble : le pays, les paysans, les paysages, Grenoble, Glénat.

9. Qui rassemble la métropole et quelques communautés de communes voisines.

10. Dont la finalité est de maintenir des pratiques agricoles adaptées ou d'encourager les changements de pratiques nécessaires pour répondre aux enjeux agri-environnementaux identifiés sur un territoire, selon la stratégie nationale : qualité de l'eau, biodiversité, maintien de prairies permanentes (http://draaf.bretagne.agriculture.gouv.fr/Les-Projets-Agroenvironnementaux)

11. Le Vinatier a toujours eu des animaux qui auparavant servaient à nourrir les habitants de l'hôpital.

12. https://www.pierrebenite.fr/3e-operation-poules-pondeuses-et-composteuses/

13. cf. Le Progrès, 3 avril 2018, "Cinq ruches, qui abriteront 250000 abeilles, vont être installées sur le toit de la gare de Jean-Macé ».

14. cf. Le Progrès, 12 décembre 2016, «Des ruches installées à Lyon pour préserver les abeilles ».

15. https://carredesoie.ucpa.com/espaces/poney-club/presentation/

16. MET, le magazine institutionnel de la Métropole de Lyon, indique dans son édition de septembre 2018 que « l'ancienne caserne Raby à Bron a commencé sa reconversion. Sur ce site de 16 hectares, un nouveau quartier va sortir de terre : dans une dizaine d'années, 2000 personnes pourront $y$ vivre, $y$ travailler, profiter des commerces et des services, dans un espace très fortement marqué par la nature. [...]. Les premiers habitants arriveront en 2021 dans les 410 premiers logements livrés, juste à côté de la grande prairie centrale ».

17. Ainsi que l'indique la délégation Auvergne-Rhône-Alpes de l'Institut français du cheval et de l'équitation : https://chevalauvergnerhonealpes.wordpress.com/2016/09/27/le-cheval-autravail-un-outil-de-developpement-durable-en-auvergne-rhone-alpes/

18. http://fermecroixrousse.fr

19. Le Progrès, 2 avril 2018, «Caluire-et-Cuire : les premières poules offertes par la ville ont été distribuées ».

20. À Pierre-Bénite, « afin d'accueillir les gallinacées dans les meilleures conditions, un éleveur professionnel assurera une formation des familles à la fin du mois de mars » (site internet municipal), tandis qu'à Caluire-et-Cuire, « les conditions [pour recevoir une poule sont d'avoir un jardin, un poulailler, [de] suivre une formation [et de] s'engager à ne pas cuisiner une poule au pot » (site internet municipal).

21. Grenoble-Alpes Métropole, 2015, L'agriculture de la Métropole. Monographie 2015, 25 p.

22. http://adabel.fr/-Ferme-de-Montgardier-.html

\section{RÉSUMÉS}

Depuis quelques années, des animaux d'élevage font leur retour dans les villes françaises, sous des formes diverses : agricoles, environnementales ou thérapeutiques. Entré par la petite porte, l'élevage en milieu urbain et périurbain reste encore méconnu. Cet article propose un tour d'horizon des places, rôles et fonctions de l'élevage dans un milieu dont il apparaît généralement 
exclu. À partir des exemples de Lyon et Grenoble, il s'agit de comprendre comment l'élevage peut être considéré comme un médium renouvelé des relations entre ville et nature, mais aussi de quelles manières il s'insère dans les nouvelles relations alimentaires entre villes et campagnes. L'article se termine par une analyse des contradictions émergentes entre types d'élevage, dans un contexte d'évolution du statut de l'animal en société.

For some years, farmed animals are back in French cities. This evolution takes agricultural, environmental or therapeutic forms. Animal husbandry remains nevertheless little known. This article offers an overview of the scopes, roles and functions of an activity which appears usually excluded from urban environments. Building on the examples of Lyon and Grenoble conurbations, our ambition is to understand how animal husbandry can be considered as a renewed medium of city/nature relationships, but also how it becomes part of the new food chains connecting cities and local rural areas. We finally analyze the emerging contradictions between various types of animal husbandry, against a backdrop marked by rapid changes in the social status of animals.

\section{INDEX}

Mots-clés : agriculture urbaine, Animal, Nature en ville, Relations villes-campagnes, Relocalisation alimentaire

Keywords : animal, food relocalisation, nature in the city, urban agriculture, urban/rural relations

\section{AUTEURS}

\section{CLAIRE DELFOSSE}

Université Lumière Lyon 2 - EA Laboratoire d'Études Rurales. 16 avenue Berthelot, 69007 Lyon, France. claire.delfosse@wanadoo.fr

\section{ADRIEN BAYSSE-LAINÉ}

Université de Cergy-Pontoise - EA MRTE. 33 boulevard du Port, 95011 Cergy-Pontoise, France / Université Lumière Lyon 2 - EA Laboratoire d'Études Rurales. 16 avenue Berthelot, 69007 Lyon, France / INRA-SAD - UMR Innovation. 2 place Pierre Viala, bât. 27, 34060 Montpellier Cedex 02, France. abl@posteo.eu 